\title{
ALTERAÇÃO DE PROPRIEDADES FÍSICAS E ATIVIDADE MICROBIANA DE UM LATOSSOLO AMARELO ÁLICO APÓS O CULTIVO COM FRUTEIRAS PERENES E MANDIOCA ${ }^{(1)}$
}

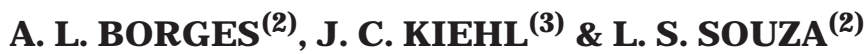

\begin{abstract}
RESUMO
Este trabalho objetivou avaliar as alterações das propriedades físicas de um Latossolo Amarelo álico (Haplorthox) franco argilo-arenoso, do município de Cruz das Almas (BA), cultivado por 15 anos com banana, 38 anos com citros, 15 anos com manga e 14 anos com mandioca, em comparação com o solo sob mata nativa. E $m$ 1991, coletaram-se amostras de solo dos horizontes $A, A B, B w_{1}$ e $\mathrm{Bw}_{2}$, determinando-se a densidade do solo, a densidade de partículas, a macroporosidade, a microporosidade, além da distribuição dos agregados em classes de tamanho e atividade microbiana. 0 cultivo do solo elevou sua densidade e reduziu a macroporosidade nas áreas cultivadas com manga e citros, notadamente no horizonte superficial. A microporosidade foi inferior no solo cultivado com manga, no horizonte superficial. A percentagem de agregados estáveis em água maiores que $0,50 \mathrm{~mm}$ foi reduzida nas glebas cultivadas com banana e manga. A atividade microbiana do solo foi mais elevada na área sob mata do que nas demais.
\end{abstract}

Termos de indexação: densidade do solo, macroporosidade, microporosidade, agregação, solo cultivado, solo natural.

(1) Recebido para publicação em setembro de 1997 e aprovado em junho de 1999.

(2) Pesquisador da E mbrapa Mandioca e Fruticultura, Caixa Postal 007, CEP 44380-000 Cruz das AImas (BA).

(3) Professor do Departamento de Ciência do Solo da Escola Superior de Agricultura Luiz de Queiroz - ESALQ-USP. Caixa Postal 9, CEP 13418-900 Piracicaba (SP). 


\title{
SUMMARY: PHYSICAL PROPERTIES AND MICROBIOLOGICAL ACTIVITY CHANGES OF AN ALIC YELLOW LATOSOL AFTER CULTIVATION WITH PERENNIAL FRUIT TREES AND CASSAVA
}

\begin{abstract}
This study aimed at evaluating changes in the physical properties of an alic sandyclay loam Yellow Latosol (Haplortox), located at Cruz das Almas, Bahia, Brazil, cropped for 15 years with banana, 38 years with citrus, 15 years with mango, and 14 years with cassava, compared with soil covered by native forest. In 1991, soil samples were coll ected for the A, $A B, B w_{1}$ and $B_{2}$ horizons and soil bulk density, soil density, macroporosity, mi croporosity weredetermined, as well as thedistribution of aggregates by sizeand mi crobi ol ogical acti vity. Soil bulk density increased and macroporosity decreased in mango and citrus areas, mainly in theupper horizon. Microporosity was lower for thesoil under mango, in theupper horizon. The percentage of stable aggregates in water larger than $0.50 \mathrm{~mm}$ decreased in the soils under banana and mango. Thesoil microbiological activity was higher in thearea covered by native forest.
\end{abstract}

Index terms: soil bulk density, soil macroporosity, soil microporosity, aggregation, cultivated soil, natural soil.

\section{INTRODUÇÃO}

As mudanças na vegetação, bem como o cultivo do solo, causam alterações nas suas propriedades físicas, pois mesmo as práticas culturais mais simples, como as capinas manuais, podem proporcionar a pulverização desua superfície (Russel \& Russel, 1961). As propriedades físicas dos solos degradam-se, geral mente, quando eles são cultivados intensamente(Skidmore et al ., 1975; J uo\& Lal, 1977; Coote \& Ramsey, 1983).

As práticas de manejo influenciam diretamente o tamanho, a distribuição e a estabilidade dos agregados do solo (Grohmann, 1960; Aina, 1979; Elliott, 1986). O diâmetro médio dos agregados será tanto menor quanto mais intensa for a movimentação do solo, provocada pelo sistema de cultivo (Grohmann \& Arruda, 1961; Aina, 1979). A redução dos níveis de matéria orgânica constitui papel fundamental na estabilização dos agregados do solo (Kononova, 1966; Allison, 1968; M bagwu \& Bazzoffi, 1988). Nos sol os sob cultivo, princi palmente nas camadas superficiais, oaumento da quantidade de mi croagregados está rel acionado com a destruição da macroestrutura pel os efeitos do preparo do sol o e do impacto da gota de chuva. Segundo Tisdall \& Oades (1982), a boa estrutura para o crescimento das plantas depende da presença de agregados de 1 a $10 \mathrm{~mm}$ de diâmetro que permanecem estáveis quando molhados. Silva (1981) constatou, em Latossolo Amarelo do sul do estado da Bahia, aumento da densidade do solo quando mecanizado em relação àquele sob vegetação de floresta, decorrente da destruição da estrutura do solo provocada pelo uso de máquina pesada.
A densidade do solo, outra propriedade física indicadora do estado de degradação do solo, também éinfluenciada pela substituição da vegetação nativa por culturas, ou seja, torna-se maior em solos cultivados (Silva, 1981; Coote\& Ramsey, 1983; Neves et al., 1991).

Cintra et al . (1983), caracterizando fisicamente o solo de um pomar de laranja 'Baianinha' submetido a diferentes práticas de manejo, verificaram que o uso contínuo e a longo prazo de grade e herbicida permitiu a formação de camadas compactadas e um adensamento na superfície. Concluíram que a prática de ceifa permanente manteve ou melhorou as propriedades físicas do solo.

Comparando as propriedades físicas do sol o sob mata e sob cultura de abacaxi, este com diferentes tempos de utilização, Cintra \& Cunha (1987) verificaram que, apesar do manejo inadequado do solo, não houve alterações significativas nas suas propriedades físicas. Constataram que os benefícios introduzi dos com a adição da matéria orgânica, pelos resíduos culturais do abacaxi, compensaram os possíveis problemas decorrentes do tempo de uso do solo e do manejo inadequado.

Além das alterações das propriedades físicas, a mudança da vegetação e as práticas culturais real izadas levam a um desequilíbrio da mi crobiota, modificando sua atividade. F onseca (1984) encontrou maior quantidade de microrganismos no solo sob eucal ipto, na camada de $0-5 \mathrm{~cm}$, do que em sol os sob mata natural e pastagem, provavelmente por causa das mudanças químicas e físicas do solo.

Os Latossolos Amarelos com horizontes subsuperficiais coesos, predominantes na região de 
Cruz das Al mas (BA), após a retirada da mata nativa, foram ocupados com vegetação secundária, culturas de subsistência e frutíferas perenes. Por serem escassos os estudos sobre as alterações em propriedades dos sol os de tabuleiro costei ro da Bahia, ainda não estão bem definidas as recomendações de uso.

Este trabalho teve como objetivo avaliar as alterações de algumas propriedades físicas e da atividade mi crobiana de um $L$ atossolo Amareloálico localizado no município de Cruz das Almas, Bahia, quando submetido a vários anos de cultivo com banana, citros, manga e mandioca.

\section{MATE RIAL E MÉTODOS}

A área estudada está situada no município de Cruz das Almas, na região do Recôncavo Baiano, no planalto pré-litorâneo, a uma latitude de 1240'19" Sul, longitude de 39006'22" WGr e a $220 \mathrm{~m}$ de altitude. A região apresenta clima subúmido, com umidade relativa do ar de $80 \%$, temperatura média anual de $24^{\circ} \mathrm{C}$ e precipitação média de $1.224 \mathrm{~mm} /$ ano (E MBRAPA, 1993). Os meses mais chuvosos são abril e maio ( $150 \mathrm{~mm} / \mathrm{mês})$, e os mais secos, setembro e outubro ( $60 \mathrm{~mm} / \mathrm{mês}$ ).

Estudou-se o Latossol o Amarel oálico, incluído na área geomórfica conhecida como tabuleiros, correspondentes aos baixos platôs costeiros, com horizonte subsuperficial coeso (EMBRAPA, 1993). Segundo a classificação textural, é um sol o francoargilo-arenoso, com horizonte superficial mais arenoso ( $740 \mathrm{~g} \mathrm{~kg}^{-1}$ areia) e com o teor de argila aumentando em profundidade.

Abriram-se três trincheiras, em 1991, em cada uma das glebas cultivadas com banana (15 anos), citros (38 anos), manga (15 anos) e mandioca (14 anos) e sob mata nativa, transição floresta subperenifólia/subcaducifólia. As gl ebas ocupavam áreas de aproximadamente um hectare ese situavam em um raio de três quilômetros, apresentando as propriedades indicadas no quadro 1 . Coletaram-se amostras dos horizontes $A(0-9 \mathrm{~cm}), A B(9-38 \mathrm{~cm})$, $\mathrm{Bw}_{1}(38-72 \mathrm{~cm})$ e $\mathrm{Bw}_{2}(72-100 \mathrm{~cm})$, de acordo com trabalho de levantamento pedológico (EMBRAPA, 1993). Na gleba cultivada com banana, os tratos culturais, como desbastee desfol ha, eram realizados periodicamente, bem como a capina manual. $\mathrm{Na}$ gleba cultivada com citros, para o controle das plantas invasoras, era feita a capina mecânica com grade, duas a três vezes durante o ano. Na gleba cultivada com manga, a superfície do solo apresentava-se coberta com um manto de fol has de aproximadamente $3 \mathrm{~cm}$ de altura.

Determinou-se a densidade do solo (ds) em amostras indeformadas, col etando-se duas repetições em cada horizonte, utilizando anéis volumétricos, conforme procedimento descrito em EMBRAPA (1979). A densidade de partículas (dp) foi determinada em amostras de solo passadas em peneira de $2 \mathrm{~mm}$ de diâmetro, col etadas ao longo do horizonte, empregando-se o método do balão volumétrico (Camargo et al., 1986).

A porosidade total $(\mathrm{Pt})$, macroporosidade e microporosidade foram analisadas conforme procedimento descrito em EMBRAPA (1979).

Avaliou-se a percentagem de agregados estáveis em água maiores que 0,5 mm (\%AG.EA) e a distribui ção dos agregados em três classes: 4,76-2,00; 2,00-1,00 e 1,00-0,50 mm, conforme EMBRAPA (1979). Além disso, foram avaliadas a quantidade de água disponível (EMBRAPA, 1979) e a atividade respiratória dos microrganismos do solo pela evolução do $\mathrm{CO}_{2}$.

Os dados obtidos foram analisados pelo teste $t$ para amostras independentes em que foram feitas comparações entre a gleba sob mata nativa com as cultivadas com banana, citros, manga e mandioca.

\section{RESULTADOS E DISCUSSÃO}

O cultivo dosolo al terou suas propriedades físicas. Noquadro 2, observa-se que, no horizontesuperficial

\section{Quadro 1. Teores de carbono, argi la, cálcio (Ca) e magnésio (Mg) no horizonte superficial e profundidade da} camada coesa de um Latossolo Amarelo álico sob diferentes coberturas vegetais

\begin{tabular}{lccccc}
\hline Cobertura vegetal & Carbono & Argila & Ca & Mg & Camada coesa \\
\hline & $\mathrm{g} \mathrm{dm}^{-3}$ & $\mathrm{~g} \mathrm{~kg}^{-3}$ & $-\mathrm{mmol}_{\mathrm{c}} \mathrm{dm}^{-3}-$ & 4 & $\mathrm{~cm}$ \\
Mata & 11,8 & 240 & 5 & 10 & $38-72$ \\
Banana & 10,4 & 230 & 20 & 8 & $17-42$ \\
Citros & 9,2 & 280 & 8 & $5-74$ \\
Manga & 6,3 & 140 & 5 & 3 & $17-50$ \\
Mandioca & 9,6 & 210 & & $9-49$ \\
\hline
\end{tabular}


(A), os solos cultivados com manga e citros apresentaram maior densidade. Sabe-se que ocultivo do solo, bem como o uso de grade, comum na gleba com citros, propiciam a formação de camadas compactadas nesse horizonte. Na gl eba com manga, provavel mente, o menor teor de carbono (Quadro 1) favoreceu o aumento da densidade.

Exceto na gleba com manga, a densidade do sol o foi maior no horizonte $A B$ (coeso nas glebas cultivadas), tendo sido no solo sob mata significativamente inferior à das demais coberturas vegetais, exceto sob citros (Quadro 2). Os sol os não cultivados, por não sofrerem ação do homem, costumam apresentar menor densidade(Silva, 1981; Coote\& Ramsey, 1983; N eves, 1991). Segundo Aina (1979), após o preparo do solo, a densidade geralmente se reduz, aumentando, posteriormente, sob a ação das chuvas durante o desenvolvimento da cultura. No horizonte $B w_{1}$, a densidade do solo diminuiu em todas as glebas, com exceção daquela sob mata; o solo cultivado com banana apresentou densidade significativamente superior à do sol o sob mata. Somente no horizonte $\mathrm{Bw}_{2}$ não houve diferenças entre os sol os cultivados e sob mata para a densidade do solo, cuja média foi de 1,44 $\mathrm{kg} \mathrm{dm}^{-3}$ (Quadro 2).

Observa-se, também no quadro 2, no solo cultivado com manga, di mi nuição da densi dade com a profundidade, mesmo considerando a presença da camada coesa de 17 a $50 \mathrm{~cm}$ da superfície.
Provavelmente, o sistema radicular pivotante da mangueira conseguiu romper esta camada coesa e melhorar a sua estrutura. Aguiar Neto \& Nacif (1988) também verificaram redução da densidade com a profundidade até o horizonte $B_{22}(+153 \mathrm{~cm})$, porém explicando o fato pelo aumento do teor de argila total em profundidade.

Apesar de o teor de argila total ter aumentado com a profundidade, foram constatadas densidades mais elevadas nos horizontes mais profundos em relação ao superficial na gl eba sob mata e mandioca (Quadro 2). Segundo Kiehl (1979), a densidade do sol o geralmente aumenta com a profundidade, pois as pressões exercidas pelas camadas superiores sobre as subjacentes favorecem a compactação. Além disso, a movimentação de material fino dos horizontes superiores para os inferiores reduz os espaços porosos, aumentando a densidade. Tal aumento pode estar relacionado também com a redução do teor de matéria orgânica.

Nos solos cultivados com mandioca, banana e citros, a densidade do sol o foi maior no horizonte AB $(9-38 \mathrm{~cm})$, onde nessas gl ebas aparece a camada coesa (Quadro 1). Tal fato ocorreu mesmo na gleba com mandioca, cuja cultura resulta em considerável revolvimento do solo na época da col heita, além do fato de o solo ser preparado anual ou bienalmente para o plantio. Da mesma maneira, em sol os tropicais da Nigéria, Hulugalle \& Ezumah (1991) não constataram influência da cultura da mandioca na

Quadro 2. Densidade do solo, macro e microporosidade no perfil de um Latossolo Amarelo álico sob diferentes coberturas vegetais

\begin{tabular}{|c|c|c|c|c|c|c|}
\hline \multirow{2}{*}{ Horizonte } & \multicolumn{5}{|c|}{ Cobertura vegetal } & \multirow{2}{*}{ Média } \\
\hline & Mata & Banana & Citros & Manga & Mandioca & \\
\hline & \multicolumn{6}{|c|}{ Densidade do solo, $\mathrm{kg} \mathrm{dm}^{-3}$} \\
\hline$A$ & 1,36 & $1,45^{*}$ & $1,57 * *$ & $1,65^{* *}$ & $1,46^{\mathrm{ns}}$ & 1,50 \\
\hline$A B$ & 1,46 & $1,62 *$ & $1,61^{\mathrm{ns}}$ & $1,60 *$ & $1,64 *$ & 1,59 \\
\hline $\mathrm{Bw}_{1}$ & 1,46 & $1,56 * *$ & $1,45^{\mathrm{ns}}$ & $1,54^{\mathrm{ns}}$ & $1,51^{\mathrm{ns}}$ & 1,50 \\
\hline \multirow[t]{2}{*}{$\mathrm{Bw}_{2}$} & 1,45 & $1,41^{\mathrm{ns}}$ & $1,40^{\text {ns }}$ & $1,41^{\mathrm{ns}}$ & $1,51^{\mathrm{ns}}$ & 1,44 \\
\hline & \multicolumn{6}{|c|}{ Macroporosidade, $\mathrm{m}^{3} \mathrm{~m}^{-3}$} \\
\hline$A$ & 0,23 & $0,21^{\text {ns }}$ & $0,15^{*}$ & $0,15^{*}$ & $0,17^{\mathrm{ns}}$ & 0,18 \\
\hline$A B$ & 0,19 & $0,08^{* *}$ & $0,08 * *$ & $0,10 * *$ & $0,11^{* *}$ & 0,11 \\
\hline$B w_{1}$ & 0,16 & $0,07^{*}$ & $0,14^{\mathrm{ns}}$ & $0,10^{\text {ns }}$ & $0,17^{\mathrm{ns}}$ & 0,13 \\
\hline \multirow[t]{2}{*}{$\mathrm{Bw}_{2}$} & 0,13 & $0,11^{\text {ns }}$ & $0,17^{\mathrm{ns}}$ & $0,18^{\text {ns }}$ & $0,14^{\mathrm{ns}}$ & 0,15 \\
\hline & \multicolumn{6}{|c|}{ Microporosidade, $\mathrm{m}^{3} \mathrm{~m}^{-3}$} \\
\hline$A$ & 0,18 & $0,17^{\mathrm{ns}}$ & $0,19^{\text {ns }}$ & $0,14^{*}$ & $0,19^{\text {ns }}$ & 0,18 \\
\hline$A B$ & 0,18 & $0,21^{*}$ & $0,24 * *$ & $0,19^{\mathrm{ns}}$ & $0,18^{\mathrm{ns}}$ & 0,20 \\
\hline$B w_{1}$ & 0,21 & $0,22^{\mathrm{ns}}$ & $0,24^{\mathrm{ns}}$ & $0,21^{\mathrm{ns}}$ & $0,17^{*}$ & 0,21 \\
\hline $\mathrm{Bw}_{2}$ & 0,23 & $0,26^{\mathrm{ns}}$ & $0,24^{\mathrm{ns}}$ & $0,20 *$ & $0,20 *$ & 0,23 \\
\hline
\end{tabular}

*, **, ns significativo a 5 e a 1\% pelo teste t e não-significativo, respectivamente, em comparação com a gleba sob mata, dentro de cada horizonte. 
densidade do sol o. No entanto, no sol o cultivado com citros, apesar de a camada coesa $(9$ a $74 \mathrm{~cm}$ ) abranger os horizontes $\mathrm{AB}$ e B $\mathrm{w}_{1}$, somente no horizonte $\mathrm{AB}$ a densidade foi mais elevada, decrescendo, posteriormente, nos horizontes mais profundos (Quadro 2). Densidades do solo el evadas reduzem os fluxos de ar, água e dos espaços vazios, aumentando a resistência mecânica dos solos e, conseqüentemente, prejudicando o desenvol vimento do sistema radicular.

A porosidadetotal de um solo, por si só, nãoéum atributo que caracteriza bem o solo, mas é a distribuição do tamanho dos seus poros, ou seja, a macroporosidade e a microporosidade.

Quanto à macroporosidade, ou porosidade de aeração, observa-se, no quadro 2, que, nos horizontes superficiais ( $A$ e AB), esta foi maior no solo sob mata do que nos sol os cultivados, com exceção do horizonte A dos solos cultivados com banana e mandioca. No horizonte $\mathrm{Bw}_{1}$, o solo cultivado com banana apresentou menor macroporosidade que o solo sob mata. Vale lembrar que a camada coesa abrangeu parte do horizonte $\mathrm{Bw}_{1}$ dessa gleba. No horizonte $\mathrm{Bw}_{2}$, as macroporosidades dos solos cultivados não diferiram das do sol o sob mata.

Para a microporosidade, observa-se, no quadro 2 que o solo cultivado com manga apresentou $22 \%$ microporos a menos que o sol o sob mata no horizonte $A$, o que pode estar relacionado com o menor teor de carbono orgânico e argila (Quadro 1). No horizonte $A B$, os solos cultivados com citros e banana apresentaram quantidade de microporos superior à do sol o sob mata. No horizonte $\mathrm{Bw}_{1}$, somente o solo cultivado com mandioca diferiu do solo sob mata, tendo apresentado valores significativamente menores, enquanto no horizonte $\mathrm{Bw}_{2}$ o sol o sob mata apresentou quantidade de microporos $1,1 \mathrm{vez}$ superior à dos solos cultivados com manga e mandioca.

Se o solo ideal é aquele que contém $1 / 3$ de macroporos (34\%) e 2/3 de microporos (66\%) (Kiehl, 1979), os dados do quadro 2 mostram, em alguns casos, situações não-ideais. No solo sob mata, a quantidade de macroporos foi superior a $40 \%$ nos horizontes $A, A B$ e $B w_{1}$, em relação à porosidade total, refletindo-se na baixa capaci dade de retenção de água; somente no horizonte $B w_{2}$, a proporção de macro e microporos aproximou-se da ideal; assim, neste caso, deduz-se que a presença do horizonte coeso $\left(\mathrm{Bw}_{1}\right.$ ) (Quadro 1) não influiu na aeração do sol o. No solo cultivado com banana, a situação foi diferente, ou seja, percebeu-se maior proporção de macroporos na superfíciee, a partir do horizonte AB, diminuiu sensivelmente a quantidade de poros de aeração, além do excesso de microporos; admitindose, portanto, uma compactação ou adensamento, já que a densidade do solo atingiu $1,62 \mathrm{~kg} \mathrm{dm}^{-3} \mathrm{em}$ razão da presença do horizonte coeso $(17-42 \mathrm{~cm})$. No solo cultivado com citros, também se observou proporção menor de macroporos no horizonte $A B$, e como a densidade do solo também foi elevada (Quadro 2), leva a crer que existe efeito da camada coesa. Tanto no sol o sob cobertura vegetal de manga quanto no sol o com mandioca, observou-se proporção de macroporos próxima à ideal no horizonte coeso (AB), podendo, então, ser considerados sol os com boa aeração ao longo do perfil do solo.

Quanto à quantidade de agregados estáveis em água maiores que $0,50 \mathrm{~mm}$, observaram-se maiores valores nos solos com mata, mandioca e citros (Quadro 3). Esperava-se, na gl eba cultivada com banana, maior percentagem de agregados estáveis em água, devido à quantidade de material orgânico restituída ao solo e à maior presença dos cátions (cálcio e magnésio) floculantes no horizonte $A$ (Quadro 1). Segundo Godefroy \& J acquin (1975), apesar de as quantidades de resíduos restituídas ao solo pelas culturas da banana e de uma floresta tropical serem as mesmas, no caso da floresta tropical há maior regularidadederestituiçãoe a temperatura do solo é baixa, o que propicia produções permanentes e mais freqüentes de substâncias húmicas, importantes para a estabilidade dos agregados.

Os solos com maior percentagem de agregados estáveis em água podem ser considerados relativamente mais resistentes à deformação e à quebra. Os tratos culturais ou mesmo o uso intensivo do solo, principalmente quando realizados de maneira incorreta, aumentam o número de agregados menores (Grohmann \& Arruda, 1961; Aina, 1979; Elliott, 1986). Cintra et al. (1983) constataram redução do tamanho dos agregados, quando foram utilizados por longo tempo herbicida e gradagem em pomar de laranja 'Baianinha'.

$\mathrm{Na}$ figura 1, observa-se a distribuição dos agregados em três classes para as diferentes coberturas vegetais e para o horizonte superficial (A), onde os efeitos foram mais evidentes. Os solos sob mata e mandioca apresentaram maiores quantidades de agregados com o diâmetro superior a 2,00 mm, embora, no caso da mandioca, o uso mais intensivo do sol o deveria contribuir para diminuir a percentagem de agregados maiores. A estabilidade dos agregados é influenciada pel o manejo e declina quando o solo é cultivado com arado e grade.

No que se refere à quantidade deágua disponível no solo, verificaram-se teores variando de 0,008 a $0,033 \mathrm{~kg} \mathrm{~kg}^{-1}$, que foram considerados baixos (Quadro 3). Vale lembrar que o solo possui em torno de $630 \mathrm{~g} \mathrm{~kg}^{-1}$ de areia. Os baixos teores de água disponível, notadamenteaté $38 \mathrm{~cm}$ de profundidade, onde se concentra grande parte do sistema radicular das plantas, são preocupantes. Práticas de manejo do solo, como irrigação e uso de cobertura morta, devem ser utilizadas a fim de aumentar ou conservar o teor de água no solo, favorecendo, assim, o desenvolvimento das culturas. 
Quadro 3. Agregados estáveis em água maiores que 0,50 mm, água disponível e atividade microbiana no perfil de um Latossolo Amarelo álico sob diferentes coberturas vegetais

\begin{tabular}{|c|c|c|c|c|c|c|}
\hline \multirow{2}{*}{ Horizonte } & \multicolumn{5}{|c|}{ Cobertura vegetal } & \multirow{2}{*}{ Média } \\
\hline & Mata & Banana & Citros & Manga & Mandioca & \\
\hline & \multicolumn{6}{|c|}{ Agregados maiores que $0,50 \mathrm{~mm}, \%$} \\
\hline A & 87,0 & $42,7 *$ & $62,0^{\text {ns }}$ & $35,6^{*}$ & $80,0^{\text {ns }}$ & 61,5 \\
\hline$A B$ & 62,9 & $39,0 *$ & $49,9^{\text {ns }}$ & $37,8^{*}$ & $52,7^{\text {ns }}$ & 48,5 \\
\hline$B w_{1}$ & 40,3 & $22,6 *$ & $47,2^{*}$ & $35,6^{\text {ns }}$ & $50,5^{\text {ns }}$ & 39,2 \\
\hline \multirow[t]{2}{*}{$\mathrm{Bw}_{2}$} & 40,6 & $24,3^{* *}$ & $48,1^{\mathrm{ns}}$ & $38,4^{\text {ns }}$ & $43,2^{\text {ns }}$ & 38,9 \\
\hline & \multicolumn{6}{|c|}{ Água disponível, $\mathbf{k g ~ k g}^{\mathbf{1}}$} \\
\hline$A$ & 0,011 & $0,014^{\mathrm{ns}}$ & $0,010^{\text {ns }}$ & $0,016^{\mathrm{ns}}$ & $0,017 *$ & 0,014 \\
\hline$A B$ & 0,017 & $0,018^{\mathrm{ns}}$ & $0,008^{\mathrm{ns}}$ & $0,023^{\mathrm{ns}}$ & $0,008^{*}$ & 0,018 \\
\hline$B w_{1}$ & 0,025 & $0,019 *$ & $0,018^{*}$ & $0,023^{\mathrm{ns}}$ & $0,023^{\mathrm{ns}}$ & 0,022 \\
\hline \multirow[t]{2}{*}{$\mathrm{Bw}_{2}$} & 0,029 & $0,023^{\mathrm{ns}}$ & $0,033^{\mathrm{ns}}$ & $0,028^{\text {ns }}$ & $0,030^{\text {ns }}$ & 0,029 \\
\hline & \multicolumn{6}{|c|}{ Atividade microbiana, $\mathrm{mg} \mathrm{CO}_{2} 100 \mathrm{~g}$ solo seco-1 } \\
\hline$A$ & 40,73 & $20,65^{*}$ & $20,18^{*}$ & $13,24^{* *}$ & $18,38^{*}$ & 22,64 \\
\hline$A B$ & 10,16 & $8,73^{\text {ns }}$ & $4,84^{\mathrm{ns}}$ & $10,63^{\text {ns }}$ & $17,04^{\mathrm{ns}}$ & 10,28 \\
\hline$B w_{1}$ & 8,77 & $4,47^{\mathrm{ns}}$ & $11,84^{\mathrm{ns}}$ & $7,04^{\mathrm{ns}}$ & $11,53^{\text {ns }}$ & 8,73 \\
\hline $\mathrm{Bw}_{2}$ & 3,48 & $1,71^{\mathrm{ns}}$ & $2,88^{\text {ns }}$ & $5,23^{\mathrm{ns}}$ & $3,80^{\mathrm{ns}}$ & 3,42 \\
\hline
\end{tabular}

*,**, ns significativo a 5 e a $1 \%$ pelo teste t e não-significativo, respectivamente, em comparação com a gleba sob mata, dentro de cada horizonte.

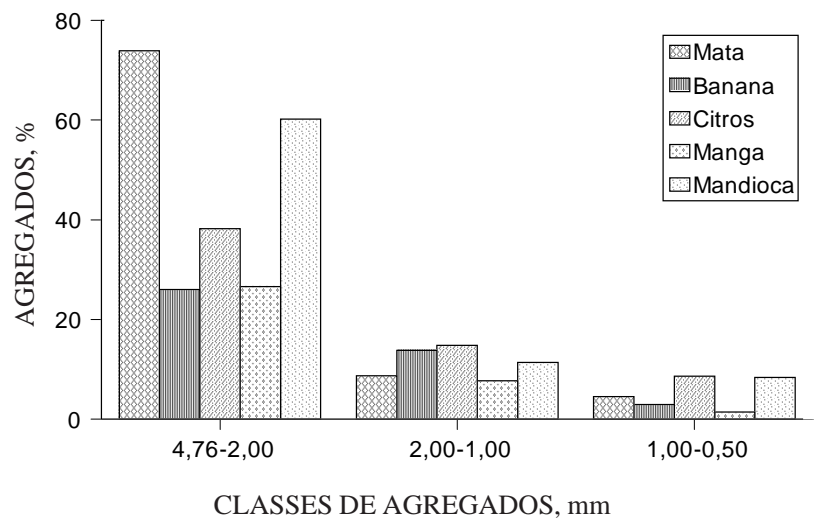

Figura 1. Distribuição de agregados (\% peso) em classes de tamanho $(\mathrm{mm})$ no horizonte superficial de um Latossolo Amarelo álico sob diferentes coberturas vegetais.

Quanto à atividade microbiana, somente no horizonte superficial houve diferenças entre as coberturas vegetais (Quadro 3). O solo sob mata revel ou atividade microbiana mais el evada do que as demais coberturas, podendotal fato ser atribuído à maior concentração de carbono na camada superior do sol o sob mata (Quadro 1). Desta maneira, ocultivo do solo, independentemente da cultura, afetou, direta ou indiretamente, a atividade pmicrobiana no
horizontesuperficial.É provável quea redução dessa atividade em profundidade esteja relacionada com a diminuição da macroporosidade (Quadro 2) e dos teores de carbono.

\section{CONCLUSÕES}

1. O cultivo do solo elevou sua densidade e reduziu a macroporosidade, notadamente no horizonte superficial $(0-9 \mathrm{~cm})$ das áreas cultivadas com manga e citros. A microporosidade foi inferior no sol o cultivado com manga nesse horizonte.

2. A percentagem deagregados estáveis emágua maiores que $0,50 \mathrm{~mm}$ na massa total do solo foi reduzida nas glebas cultivadas com banana emanga.

3. A atividade mi crobiana do sol o foi mais elevada na área sob mata do que nas demais, apenas no horizonte superficial.

\section{LITE RATURA CITADA}

AGUIAR NETTO, A.O. \& NACIF, P.G.S. Caracterização morfológica e físico-hídrica de solos representativos do Recôncavo Baiano. II - Determinação da capacidade de campo "in situ" e suas relações com dados obtidos em laboratório. Cruz das Almas, Universidade Federal da Bahia, 1988. 58p. 
AI NA, P.O. Soil changes resulting from long-term management practices in Western Nigeria. Soil Sci. Soc. Am. J ., 43:173177, 1979.

ALLISON, F.E. Soil aggregation - Some facts and fallacies as seen by a microbiologist. Soil Sci., 106:136-143, 1968.

CAMARGO, O.A.; MONIZ, A.C.; J ORGE, J .A. \& VALADARES, J .M.A.S. Métodos de análise química, mineralógica e física de sol os do Instituto Agronômico de Campinas. Campinas, Instituto Agronômico, 1986. 94p. (Boletim Técnico, 106)

CINTRA, F.L.D. \& CUNHA, G.A.P. Caracterizaçãofísica de solos cultivados com abacaxi em áreas com diferentes tempos de utilização. R. Bras. Frut., 9:7-15, 1987.

CINTRA, F.L.D.; COELHO, Y.S.; CUNHA SOBRINHO, A.P. \& PASSOS, O.S. Caracterização física do solo submetido a prática de manejo em pomar de laranja 'Baianinha'. Pesq. Agropec. Bras., 18:173-179, 1983.

COOTE, D.R. \& RAMSEY, J .F. Quantification of the effects of over 35 years of intensive cultivation on four soils. Can J . Soil Sci., 63:1-14, 1983.

ELLIOTT, E.T. Aggregate structure and carbon, nitrogen, and phosphorus in native and cultivated soils. Soil Sci. Soc. Am. J ., 50:627-633, 1986.

EMPRESA BRASILEIRA DE PESQUISA AGROPECUÁRIA EMBRAPA. Serviço Nacional de Levantamento e Conservação de Solos. Manual de métodos de análises de solo. Rio de J aneiro, 1979. não paginado.

EMPRESA BRASILEIRA DE PESQUISA AGROPECUÁRIA EMBRAPA. Centro Nacional de Pesquisa de Mandioca e Fruticultura Tropical. Levantamento detalhado dos solos do Centro Nacional de Pesquisa de Mandioca eF ruticultura Tropical, Cruz das Almas, Bahia. Rio deJ aneiro, 1993. 126p. (EMBRAPA.CNPMF. Boletim de Pesquisa, 7)

FONSECA, S. Propriedades físicas, químicas e microbiológicas de um Latossolo Vermelho-Amarelo sob eucalipto, mata natural epastagem. Viçosa, U niversidade F ederal deViçosa, 1984. 78p. (Tese de Mestrado)

GODEFROY, J. \& J ACQUIN, F. Relation entre la stabilité structurale des sols cultivés et les apports organiques en conditions tropicales; comparasion avec les sols forestiers. Fruits, 30:595-612, 1975.
GROHMANN, F. Análise de agregados de solos. Bragantia, 19:201-213. 1960.

GROHMANN, F. \& ARRUDA, H.V. Influência do preparo do solo sobre a estrutura da Terra-Roxa-Legítima. Bragantia, 20:1203-1209, 1961.

HULUGALLE, N.R. \& EZUMAH, H.C. Effects of cassava based cropping systems on physico-chemical properties of soil and earthworm casts in a tropical Alfisol. Agr. E cosys. Environ., 35:55-63, 1991.

JUO, A.S.R. \& LAL, R. The effect of fallow and continuous cultivation on the chemical and physical properties of an Alfisol in Western Nigeria. Plant Soil, 47:567-584, 1977.

KIEHL, E.J . Manual de edafologia - relações solo-planta. São Paulo, Agronômica Ceres, 1979. 264p.

KONONOVA, M.M. Soil organic matter, its nature, its role in soil formation and in soil fertility. 2.ed. New York, Pergamon Press, 1966. 544p.

MBAGWU, J S.C. \& BAZZOFFI, P. Stability of microaggregates as influenced by antecedent moisture content, organic waste amendment and wetting and drying cycles. Catena, 15:565576, 1988.

NEVES, A.D.S.; LIMA, F.A.M. \& MOREIRA, E.G.S. Efeito de coberturas vegetais sobre propriedades físicas de L atossolo Amarelo (Pará). In: CONGRESSO BRASILEIRO DE CIÊNCIA DO SOLO, 23., Porto Alegre, 1991. Resumos. Porto Alegre, SBCS/UFRGS, 1991. p.169.

RUSSEL, E.J . \& RUSSEL, R.W. Soil conditions and plant growth. London, Longman \& Green, 1961. 770p.

SILVA, L.F. Alterações edáficas em "solos de tabuleiro" (Haplorthoxs) por influência do desmatamento, queima e sistemas de manejo. R. Theobroma, 11:5-9, 1981.

SKIDMORE, E.L.; CARSTENSON, W.A. \& BANBURY, E.E. Soil changes resulting from cropping. Soil Sci. Soc. Am. Proc., 39:964-967, 1975.

TISDALL, J.M. \& OADES, J.M. Organic matter and water-stable aggregates in soils. J. Soil Sci., 33:141-163, 1982. 